\title{
Hypothalamic response to the chemo-signal androstadienone in gender dysphoric children and adolescents
}

\author{
Sarah M. Burke ${ }^{1,2}{ }^{*}$, Peggy T. Cohen-Kettenis ${ }^{1}$, Dick J. Veltman ${ }^{3}$, Daniel T. Klink ${ }^{4}$ and Julie Bakker ${ }^{1,2,5}$ \\ Center of Expertise on Gender Dysphoria, Neuroscience Campus Amsterdam, Department of Medical Psychology, VU University Medical Center, Amsterdam, \\ Netherlands \\ ${ }^{2}$ Neuroendocrinology Group, Netherlands Institute for Neuroscience, Amsterdam, Netherlands \\ ${ }^{3}$ Department of Psychiatry, Neuroscience Campus Amsterdam, VU University Medical Center, Amsterdam, Netherlands \\ ${ }^{4}$ Center of Expertise on Gender Dysphoria, Department of Pediatric Endocrinology, VU University Medical Center, Amsterdam, Netherlands \\ ${ }^{5}$ GIGA Neuroscience, University of Liege, Liege, Belgium
}

\section{Edited by:}

Hubert Vaudry, University of Rouen,

France

Reviewed by:

Alexander S. Kauffman, University of

California San Diego, USA

Hubert Vaudry, University of Rouen,

France

*Correspondence:

Sarah M. Burke, Center of Expertise on Gender Dysphoria, Neuroscience Campus Amsterdam, Department of Medical Psychology, VU University Medical Center, De Boelelaan 1131, Amsterdam 1081 HX, Netherlands e-mail: s.burke@vumc.nl
The odorous steroid androstadienone, a putative male chemo-signal, was previously reported to evoke sex differences in hypothalamic activation in adult heterosexual men and women. In order to investigate whether puberty modulated this sex difference in response to androstadienone, we measured the hypothalamic responsiveness to this chemo-signal in 39 pre-pubertal and 41 adolescent boys and girls by means of functional magnetic resonance imaging. We then investigated whether 36 pre-pubertal children and 38 adolescents diagnosed with gender dysphoria (GD; DSM-5) exhibited sex-atypical (in accordance with their experienced gender), rather than sex-typical (in accordance with their natal sex) hypothalamic activations during olfactory stimulation with androstadienone. We found that the sex difference in responsiveness to androstadienone was already present in pre-pubertal control children and thus likely developed during early perinatal development instead of during sexual maturation. Adolescent girls and boys with GD both responded remarkably like their experienced gender, thus sex-atypical. In contrast, pre-pubertal girls with GD showed neither a typically male nor female hypothalamic activation pattern and pre-pubertal boys with GD had hypothalamic activations in response to androstadienone that were similar to control boys, thus sex-typical. We present here a unique data set of boys and girls diagnosed with GD at two different developmental stages, showing that these children possess certain sex-atypical functional brain characteristics and may have undergone atypical sexual differentiation of the brain.

Keywords: androstadienone, chemo-signal, fMRI, gender dysphoria, hypothalamus, puberty, sex difference

\section{INTRODUCTION}

In humans, the odorous steroid 4,16-androstadien-3-one (androstadienone) has been studied intensively as a putative male modulator chemo-signal. Androstadienone, probably synthesized in the gonads (1), is secreted by the apocrine glands and can be found on the skin surface and axillary hairs (2), as well as in several body fluids including sweat and semen $(3,4)$. Higher concentrations of androstadienone in sweat have been found in men compared to women $(5,6)$. Exposure to androstadienone has been shown to affect women's mental state (7-10), and to elicit physiological responses in a sex-dependent manner (7, 9, 11-15). Moreover, smelling androstadienone had an impact on women's hormone levels (16) and affected their behavioral responses dependent on the phase of the menstrual cycle (17).

In line with the effects androstadienone had on the autonomic nervous system and on behavior, studies conducted by Savic et al. (18), using positron emission tomography (PET), showed that smelling androstadienone induced a response in the hypothalamus of heterosexual women but not in heterosexual men. The latter only showed an activation in brain areas belonging to the main olfactory system, such as the piriform cortex and amygdala (18). Thus, olfactory stimulation with androstadienone offers a relatively simple and objective experimental procedure for investigating functional sex differences in the human brain. Using functional magnetic resonance imaging (fMRI), we recently replicated the finding of women showing a stronger hypothalamic activation than men, after smelling the highest $(10 \mathrm{mM})$ out of three concentrations tested (19).

Secretion of androstadienone increases substantially during puberty. As a result, sex-related changes in olfactory sensitivity to androstenes have been reported during adolescence (20-23) with male adolescents exhibiting more anosmia to androstadienone, and an increase of odor threshold with age, compared to female adolescents. It may be inferred that the decrease in sensitivity to odorous steroids in pubescent boys is related to their increased production of endogenous androgens during puberty, probably reflecting adaptation toward (male) body odors.

Sisk and colleagues $(24,25)$ proposed a two-stage model of sexual differentiation of brain and behavior, in which early perinatal organizational effects of steroid hormones are followed by a second 
steroid-dependent sensitive phase of neurodevelopment during adolescence. Thus, puberty may be considered an organizational period in itself, in which sex differences in brain morphology and function are established or consolidated. Therefore, our first aim was to investigate whether the hypothalamic response to androstadienone develops during puberty, as part of sexual maturation, or whether it would already be present in pre-pubertal children and thus established during early brain development. Thus, we tested whether the response to androstadienone varied as a function of sex and pubertal status in four groups of pre-pubertal and adolescent boys and girls.

Of note, further studies by Savic et al. (26-28) showed that hypothalamic activation upon exposure to androstadienone also depended on sexual orientation $(27,28)$ and gender identity. Adult men diagnosed with gender dysphoria (GD) [DSM-5, Ref. (29)], and thus having a female gender identity showed a sex-atypical hypothalamic response, which reflected more on their experienced gender than their natal sex (26). This is in line with the hypothesis that individuals, diagnosed with GD might have undergone a sex-atypical programing of the nervous system $(30,31)$. The etiology of GD is currently unknown, however, a multitude of factors, ranging from adverse psycho-dynamic parent-child interactions (32), an anxious personality predisposition (33), genetic risk factors (34), and exposure to atypical levels of perinatal sex steroids during a critical period of sexual differentiation of the brain (35) have all been proposed to facilitate the development of GD.

At the Center of Expertise on Gender Dysphoria in Amsterdam, the current treatment protocol allows adolescents diagnosed with GD that persisted from childhood into adolescence to start treatment with gonadotropin-releasing hormone analogs ( $\mathrm{GnRHa}$ ) from the age of 12 years, to suppress endogenous gonadal stimulation and thus the development of irreversible sex characteristics of the natal sex (36). From the age of 16 years on, as a first step in sex reassignment they receive cross-sex hormone treatment, i.e., biological boys receive estrogens and biological girls receive androgens $(37,38)$.

A second aim of the current study was therefore to explore whether children and adolescents, diagnosed with GD, would show brain responses that reflect their expressed/experienced gender rather than their natal sex, and whether these would vary as a function of their developmental phase. Four groups of subjects, all diagnosed with GD, participated in the current study: girls and boys that were pre-pubertal and treatment-naïve, and girls and boys that were adolescent in age (though in hypogonadal state due to GnRHa treatment). None of our participants received cross-sex hormones at the time of data acquisition.

\section{MATERIALS AND METHODS \\ SUBJECTS}

The initial study sample consisted of a total of 158 participants. Four subjects were excluded from further analysis, because of anatomical anomalies (one adolescent), technical errors during data collection (one child), or because the diagnosis GD had been revised since their participation in the study (two boys with GD in remission). All participants diagnosed with GD, were recruited via the Center of Expertise on Gender Dysphoria at the VU University Medical Center in Amsterdam. The control participants were recruited via several primary and secondary schools in the Netherlands and by inviting friends and relatives of the participants with GD.

The children sample consisted of 19 control girls [mean years of age $(M)=9.7$, standard deviation $(\mathrm{SD})=0.9$ ], 20 control boys $(M=9.5, \mathrm{SD}=1.1), 17$ girls with $\mathrm{GD}(M=9.6, \mathrm{SD}=1.1)$, and 19 boys with $\mathrm{GD}(M=10.4, \mathrm{SD}=0.9)$. All children underwent a short physical examination by a pediatric endocrinologist (Daniel T. Klink) in order to ascertain their pre-pubertal status (Tanner stage 1) $(39,40)$.

The adolescent groups consisted of 21 control girls $(M=16.3$, $\mathrm{SD}=0.9), 20$ control boys $(M=15.0, \mathrm{SD}=0.6), 21$ girls with GD $(M=16.1, \mathrm{SD}=0.8)$, and 17 boys with $\mathrm{GD}(M=15.3$, $\mathrm{SD}=1.2)$. The adolescent participants, diagnosed with GD, had been treated monthly with $3.75 \mathrm{mg}$ of Triptorelin (Decapeptyl$\mathrm{CR}^{\circledR}$, Ferring, Hoofddorp, the Netherlands) by injection for on average 24 months (range 2-48 months), resulting in complete suppression of gonadal hormone production. Female adolescent controls were tested randomly according to their menstrual cycle and 11 out of 21 control girls reported using hormonal contraception.

\section{ASSESSMENTS AND SUBJECT CHARACTERISTIC}

Sexual orientation was difficult to assess, especially in the prepubertal sample, because most children were simply too young to be able to report their sexual orientation. Therefore, current or presumed future sexual attraction was assessed by asking whether the participant had ever been in love with somebody, and if yes, whether that person was a boy or a girl. Normal olfactory function was ascertained by means of an extended version of the “Sniffin' Sticks" test battery (32-item odor identification test and olfactory threshold measurement) (41-43). Furthermore, participants were asked to report the perceived intensity of a $10 \mathrm{mM}$ androstadienone solution (on a scale from 0 to 10). Separately, for the adolescent and the children samples, one-way analyses of variance (ANOVA) were conducted, using the Statistical Package for the Social Sciences, version 20 (SPSS Inc., Chicago, IL, USA), testing whether the four gender groups differed on any of these measures. Bonferroni correction for multiple comparisons was applied for post hoc tests, considering a threshold of $p<0.05$ as statistically significant. All subjects and their legal guardians gave their informed consent according to the Declaration of Helsinki, and the study was approved by the Ethics Committee of the VU University Medical Center Amsterdam (application number NL31283.029.10).

\section{OLFACTORY STIMULATION}

Androstadienone (Steraloids Inc., Newport, RI 02840, USA) was diluted in propylene glycol (Sigma) to a concentration of $10 \mathrm{mM}$, according to the "high" concentration used in our previous study (19). The volume of the solution used during the fMRI experiments was $20 \mathrm{ml}$. Olfactory stimuli were delivered through a tubing system to the subjects' nostrils by means of a custom-built airdilution olfactometer [for details of the olfactometer set-up and procedure see Ref. (19)]. With a total air flow of about $1 \mathrm{~L} / \mathrm{min}$, during "ON" periods every $2 \mathrm{~s}$, the odor was delivered during $1 \mathrm{~s}$, while during "OFF" periods subjects received odorless air. 


\section{IMAGE ACOUISITION}

Scans were performed on a 3.0-T GE Signa HDxt scanner (General Electric, Milwaukee, WI, USA). A gradient echo, echo planar imaging sequence was used for functional imaging $\left(19.2 \mathrm{~cm}^{2}\right.$ field of view, TR of $1950 \mathrm{~ms}$, TE of $25 \mathrm{~ms}$, an $80^{\circ}$ flip angle, isotropic voxels of $3 \mathrm{~mm}$, and 36 slices). Before each imaging session, a local high-order shimming technique was used to reduce susceptibility artifacts. A scanning session consisted of six alternating ON-OFF cycles over 108 volumes in a classical block design (one block consisted of nine volumes), lasting $3.6 \mathrm{~min}$. For co-registration with the functional images, a T1-weighted scan was obtained (3D FSPGR sequence, $25 \mathrm{~cm}^{2}$ field of view, TR of $7.8 \mathrm{~ms}$, TE of $3.0 \mathrm{~ms}$; slice thickness of $1 \mathrm{~mm}$, and 176 slices).

\section{IMAGE PROCESSING}

Data analysis was performed with SPM8 software (Statistical Parametric Mapping; Wellcome Department of Imaging Neuroscience, Institute of Neurology at the University College London, UK) implemented in Matlab R2009b (Math Works Inc., Natick, MA, USA). Functional images were slice-timed and realigned to the mean image, followed by unwarp. Applying the "New Segment" and "Create Template" options of the DARTEL (Diffeomorphic Anatomical Registration Through Exponentiated Lie Algebra) toolbox, structural images were segmented. Then, gray matter and white matter images were used for creating age-group specific templates (one for the children and the adolescents sample each), registered in Montreal neurological institute (MNI) space. Functional images were spatially normalized to their respective group-template, applying each individual's DARTEL flow field, and finally, images were smoothed by means of a $5-\mathrm{mm}$ full width half maximum (FWHM) isotropic Gaussian kernel.

Individual image data were analyzed using boxcar regressors convolved with a synthetic hemodynamic response function and a first-order time-modulation (TM) regressor to test for possible effects of adaptation/sensitization to androstadienone. In order to account for assumed late "wash-out" effects during OFF blocks and an early peak response to the odor stimulation during ON blocks, first-level contrast images were built by subtracting the second half (b) of the OFF blocks (four volumes) from the first part (a) of the ON blocks (four volumes). Accordingly, this was done with the associated TM regressor blocks. Further, based on the image realignment process, individual head jerks were identified (>1 mm displacement) (44). Together with the six motion parameters, these so-called scan nulling regressors were included in every first-level design matrix to account for the effects of excessive head motion.

\section{STATISTICAL ANALYSES}

First, in order to test whether the sex difference in response to androstadienone was present in both developmental control groups, and whether that sex difference in responsiveness varied as a function of adaptation/sensitization to the odor, we conducted a sex (control boys, control girls) by odor stimulation ANOVA, for both the pre-pubertal and the adolescent control groups. The factor odor stimulation consisted of two levels, a regressor modeling the condition $\mathrm{ON}_{\mathrm{a}}-\mathrm{OFF}_{\mathrm{b}}$ effect, thus the hypothesized hypothalamic response to the odor, and a first-order parametric modulation $\mathrm{TM} \mathrm{ON} \mathrm{N}_{\mathrm{a}}-\mathrm{TM} \mathrm{OFF}_{\mathrm{b}}$ regressor, which signifies how well the hypothalamic response correlates with changes over time, thus modeling possible effects of adaptation or sensitization.

Second, by means of four gender by odor stimulation ANOVAs separately for the pre-pubertal and the adolescent groups, we tested whether boys and girls diagnosed with GD differed significantly in response to the steroid odor in comparison to their respective natal sex or their experienced gender control group.

Analyses were restricted to the hypothalamus area as region of interest (ROI), defined [with Marsbar (45)] as a sphere (centered at MNI coordinates $x=0, y=-10, z=-7$; with a $7-\mathrm{mm}$ radius), and based on anatomical demarcations following (46) and (47). The threshold for statistical significance was set at $p<0.05$ family-wise error (FWE)-corrected for the extent of the hypothalamus ROI.

\section{RESULTS}

\section{PSYCHOPHYSICS AND SUBJECT CHARACTERISTICS}

Demographic, self-report, and subject characteristics are presented in Table 1. The adolescent boys with GD were significantly younger and less physically mature than the three other adolescent groups. The pre-pubertal boys with GD were significantly older than the pre-pubertal control boys. Therefore, in all group comparisons involving boys with GD, age was included as covariate. As expected, both adolescent control groups differed significantly from the boys and girls diagnosed with GD with respect to reported sexual orientation. There were no group differences in olfactory performance and on ratings of the perceived intensity of androstadienone.

\section{SEX DIFFERENCES IN HYPOTHALAMIC ACTIVATION - EFFECTS OF PUBERTY}

In order to determine whether hypothalamic activation upon smelling androstadienone is dependent on puberty, we conducted separate ANOVAs in the pre-pubertal and the adolescent groups, and compared hypothalamic activation in control girls to that of control boys during exposure to androstadienone. Results are displayed in Table 2.

Pre-pubertal girls and boys did not differ in terms of general hypothalamic responsiveness (condition effect $\mathrm{ON}>\mathrm{OFF}$ ) to androstadienone, but directional $t$-tests showed that the sex difference (girls $>$ boys) was significantly modulated by the effect of the TM regressor $(t=3.3 ; p=0.033)$, indicating that hypothalamic activation in boys differed from that of the girls during the course of the scanning session, thus suggesting differences in adaptation and/or sensitization to the odor between groups (see Figures 1A and $2 \mathrm{~A}, \mathrm{C}$ ).

Accordingly, similar comparisons were done in the adolescent groups. Again, the sex difference in hypothalamic activation (girls $>$ boys) was significantly dependent on the factor time $(t=3.5 ; p=0.019)$ (see Figures 1B and 3A,D). Visual inspection of the data (see Figure 1) revealed that control girls' activation increased with repeated exposure to androstadienone (sensitization), particularly toward the end of the session, whereas control boys' responsiveness to the steroid odor seemed relatively stable, showing a slight decrease in activation during the course of the stimulation session. 


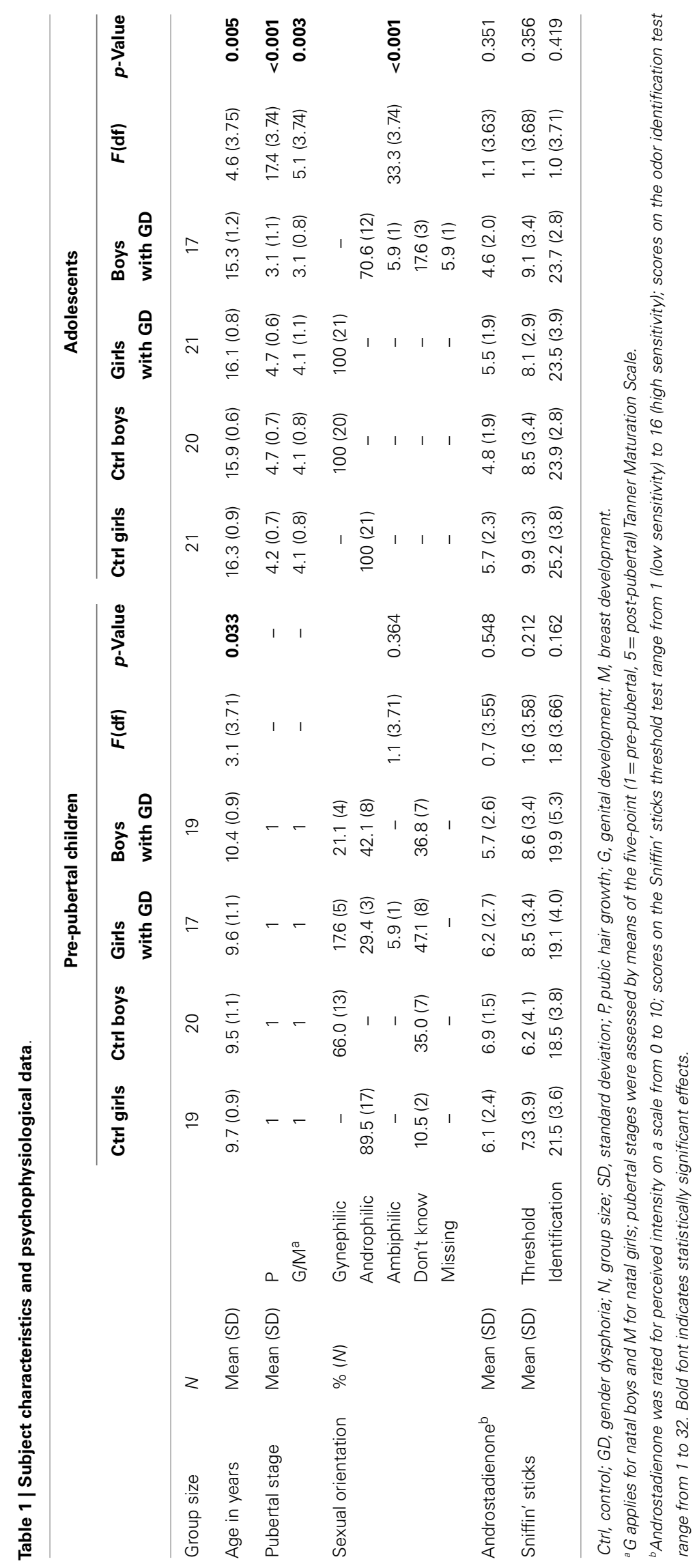


Table 2 | ANOVA results showing sex differences in hypothalamus activations in the control groups, and sex-atypical hypothalamic activations, reflecting their experienced gender, in individuals with GD.

\begin{tabular}{llrrrrrr}
\hline & Effect & $\boldsymbol{Z}_{\max }$ & $\boldsymbol{x}$ & $\boldsymbol{y}$ & $\boldsymbol{z}$ & $\boldsymbol{N}$ & $\boldsymbol{P}_{\text {FWE }}$ \\
\hline \multicolumn{1}{l}{ CTRL GIRLS } & C CTRL BOYS & & & & & & \\
Children & Gender & 2.5 & -6 & -12 & -5 & 30 & 0.202 \\
& Odor & 2.4 & -2 & -15 & -2 & 39 & 0.244 \\
& ON-OFF & 1.8 & 0 & -10 & -14 & 1 & 0.456 \\
& ONTM-OFFTM & 3.2 & -6 & -12 & -5 & 92 & $\mathbf{0 . 0 3 3}$ \\
& & 2.9 & 6 & -7 & -8 & & \\
Adolescents & Gender & 1.7 & -3 & -7 & -2 & 17 & 0.091 \\
& Odor & 1.7 & 6 & -7 & -6 & 8 & 0.655 \\
& ON-OFF & 3.4 & 6 & -7 & -8 & 1 & 0.522 \\
& ONTM-OFFTM & 2.5 & -6 & -12 & -5 & 115 & $\mathbf{0 . 0 1 9}$
\end{tabular}

\begin{tabular}{|c|c|c|c|c|c|c|c|}
\hline \multicolumn{8}{|c|}{ CTRL GIRLS > GIRLS WITH GD } \\
\hline Children & No effects & & & & & & \\
\hline \multirow[t]{4}{*}{ Adolescents } & Gender & 3.5 & 6 & -7 & -9 & 76 & 0.016 \\
\hline & Odor & 1.7 & -6 & -7 & -6 & 1 & 0.627 \\
\hline & ON-OFF & - & - & - & - & - & - \\
\hline & $\mathrm{ON}_{\mathrm{TM}}-\mathrm{OFF} \mathrm{F}_{\mathrm{TM}}$ & 4.1 & 6 & -7 & -9 & 214 & 0.002 \\
\hline \multicolumn{8}{|c|}{ BOYS WITH GD > CTRL BOYS } \\
\hline \multirow[t]{5}{*}{ Children } & Gender & 1.8 & 2 & -14 & -2 & 4 & 0.447 \\
\hline & Odor & 1.9 & 0 & -10 & 0 & 3 & 0.436 \\
\hline & ON-OFF & 1.7 & -4 & -6 & -4 & 12 & 0.437 \\
\hline & $\mathrm{ON}_{\mathrm{TM}}-\mathrm{OFF} \mathrm{F}_{\mathrm{TM}}$ & 1.9 & 0 & -10 & -12 & 31 & 0.329 \\
\hline & & 2.4 & 0 & -14 & -12 & & \\
\hline \multirow[t]{4}{*}{ Adolescents } & Gender & 2.2 & 6 & -8 & -6 & 11 & 0.237 \\
\hline & Odor & 3.0 & 0 & -14 & -12 & 1 & 0.341 \\
\hline & ON-OFF & 1.8 & 2 & -14 & -2 & 36 & 0.046 \\
\hline & $\mathrm{ON}_{T M}-\mathrm{OFF}_{\mathrm{TM}}$ & - & - & - & - & - & - \\
\hline
\end{tabular}

$\begin{array}{llcccccc}\text { BOYS WITH GD }>\text { GIRLS WITH GD } & & & & & \\ \text { Children } & \text { No effects } & & & & & & \\ \text { Adolescents } & \text { Gender } & - & - & - & - & - & - \\ & \text { Odor } & 1.9 & 3 & -12 & -9 & 12 & 0.535 \\ & \text { ON-OFF } & - & - & - & - & - & - \\ & \text { ONTM-OFF TM } & 2.1 & 3 & -10 & -2 & 26 & 0.323\end{array}$

Ctrl, control; GD, gender dysphoria; $Z_{\max }, z$-value of peak activation voxel; $x$ y $z$, coordinates in Montreal Neurological Institute (MNI) space; N, number of clustering voxels (cluster size); FEW, family-wise error; the effects "Gender" and "Odor" indicate omnibus F-contrasts for the factors gender and odor stimulation; "ONOFF" and "ON ${ }_{T M}-O F F_{T M}$ " indicate directional t-contrasts for the condition effect and the first-order time-modulation regressor, respectively. Bold font indicates statistically significant effects.

\section{GENDER IDENTITY AND HYPOTHALAMIC ACTIVATION}

In order to test whether individuals diagnosed with GD would show a hypothalamic activation that reflected their experienced gender, and whether this brain response would be different for prepubertal and adolescent subjects, four ANOVAs were conducted, in which control girls were compared to girls with GD, and control boys were compared to boys with GD (see Table 2). In addition, we investigated the reverse effects, i.e., whether individuals with GD showed sex-typical activations, and thus deviated from their experienced gender by comparing girls with GD to control boys, and boys with GD to control girls (see Table 3). Comparing girls with GD to boys with GD revealed no significant differences, thus the sex difference in response to androstadienone observed in the control groups was absent in the groups diagnosed with GD.

\section{Girls diagnosed with GD}

None of the comparisons between pre-pubertal control girls and pre-pubertal girls with GD revealed any differences in hypothalamic activation upon smelling androstadienone. However, no sextypical effects (i.e., a female-typical hypothalamus response), when compared to control boys, could be confirmed. Thus, pre-pubertal girls with GD neither differed significantly from their experienced (control boys) nor from their natal sex (control girls) in terms of hypothalamic activation when smelling androstadienone.

In contrast, the comparison of adolescent control girls to girls diagnosed with GD revealed a significant effect of gender (control girls > girls with GD), which was mainly explained by the effect of the TM regressor $(t=4.3 ; p=0.002)$ (see Table 2; Figures 3B,E). Thus, control girls showed a significantly stronger hypothalamic response to androstadienone over time as described earlier, whereas the activation in girls with GD, similar to adolescent control boys, remained stable throughout the scanning session. The reverse group comparisons (girls with GD > control boys) revealed no significant effects (see Figure 3F), indicating that adolescent girls with GD showed no hypothalamic activation upon smelling androstadienone as was observed in adolescent control boys.

\section{Boys diagnosed with GD}

No significant effects were revealed when comparing pre-pubertal boys with GD with control boys. In contrast, a significant effect of gender, driven by the TM regressor $(t=3.5$; $p=0.013$ FWEcorrected) was revealed when comparing pre-pubertal boys with GD to control girls, indicating that pre-pubertal boys with GD showed a pattern of hypothalamic activation that was similar to that of the pre-pubertal control boys (see Table 3; Figures 2B,D,E).

When adolescent boys with GD were compared to adolescent control boys we observed a significant effect of condition $(\mathrm{ON}>\mathrm{OFF})(t=1.8 ; p=0.046)$ (see Table 2; Figures 3C,G,H). Thus, adolescent boys with GD showed a significantly stronger, thus sex-atypical response to androstadienone compared to control boys, irrespective of the factor time. The reverse contrast (control girls $>$ adolescent boys with GD), i.e., testing whether adolescent boys with GD would show an activation according to their natal sex, revealed no significant activations. Thus, adolescent boys with GD showed female-typical hypothalamic responses upon smelling androstadienone without, however, any effects due to sensitization.

\section{DISCUSSION}

\section{SEX DIFFERENCES IN HYPOTHALAMIC ACTIVATION - EFFECTS OF PUBERTY}

The present study is, to our knowledge, the first to demonstrate that sex differences in hypothalamic activation upon smelling androstadienone are already present before puberty, and thus may be considered as a sex difference established during early brain development. We found that pre-pubertal as well as 

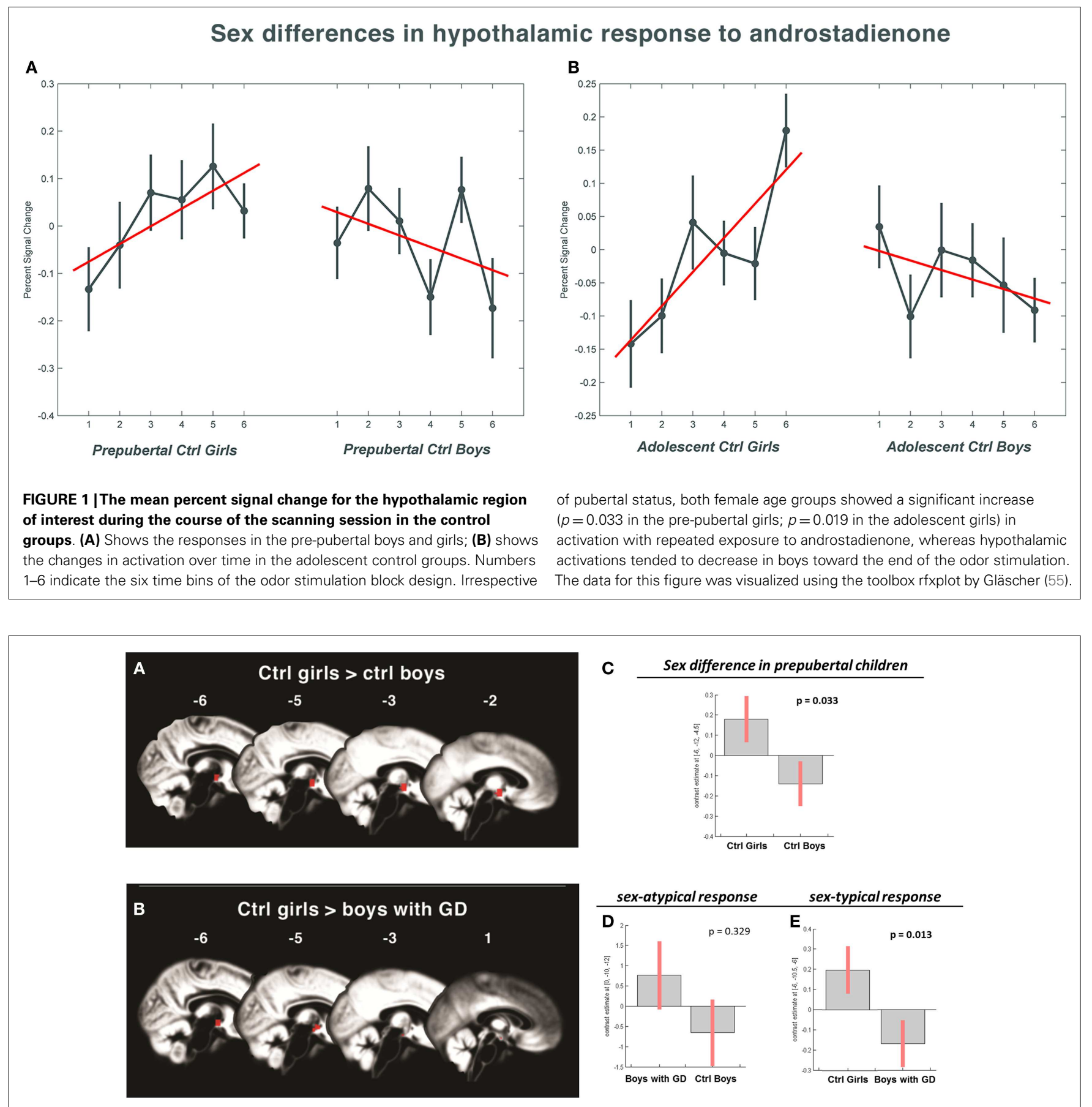

FIGURE 2 | (A,B) Show the anatomic locations of the significant hypothalamic activations, indicated in red, in response to the chemo-signal androstadienone in the pre-pubertal age groups. The numbers above each sagittal plane represent the $x$-axis coordinates in Montreal Neurological Institute space. All voxels within the hypothalamic region of interest, surviving the statistical threshold of $p<0.05$ (FWE-corrected) are shown.
(C-E) Show the bar graphs of the corresponding group contrasts for the first-order time-modulation regressor, indicating group differences in sensitization to the steroid odor. (C) Displays the sex difference, i.e., between control girls and boys; (D) shows the non-significant sex-atypical (i.e., female-like) and (E) indicates the significant sex-typical (i.e., male-like) response of boys with gender dysphoria. adolescent control girls showed a stronger hypothalamic activation compared to boys (at both developmental stages), and that this sex difference was crucially modulated by effects of sensitization to androstadienone.
Previous psychophysiological studies in children and adolescents have shown that olfactory sensitivity to androgenic odors differed between boys and girls during development, due to the increased production of endogenous androgens by boys 

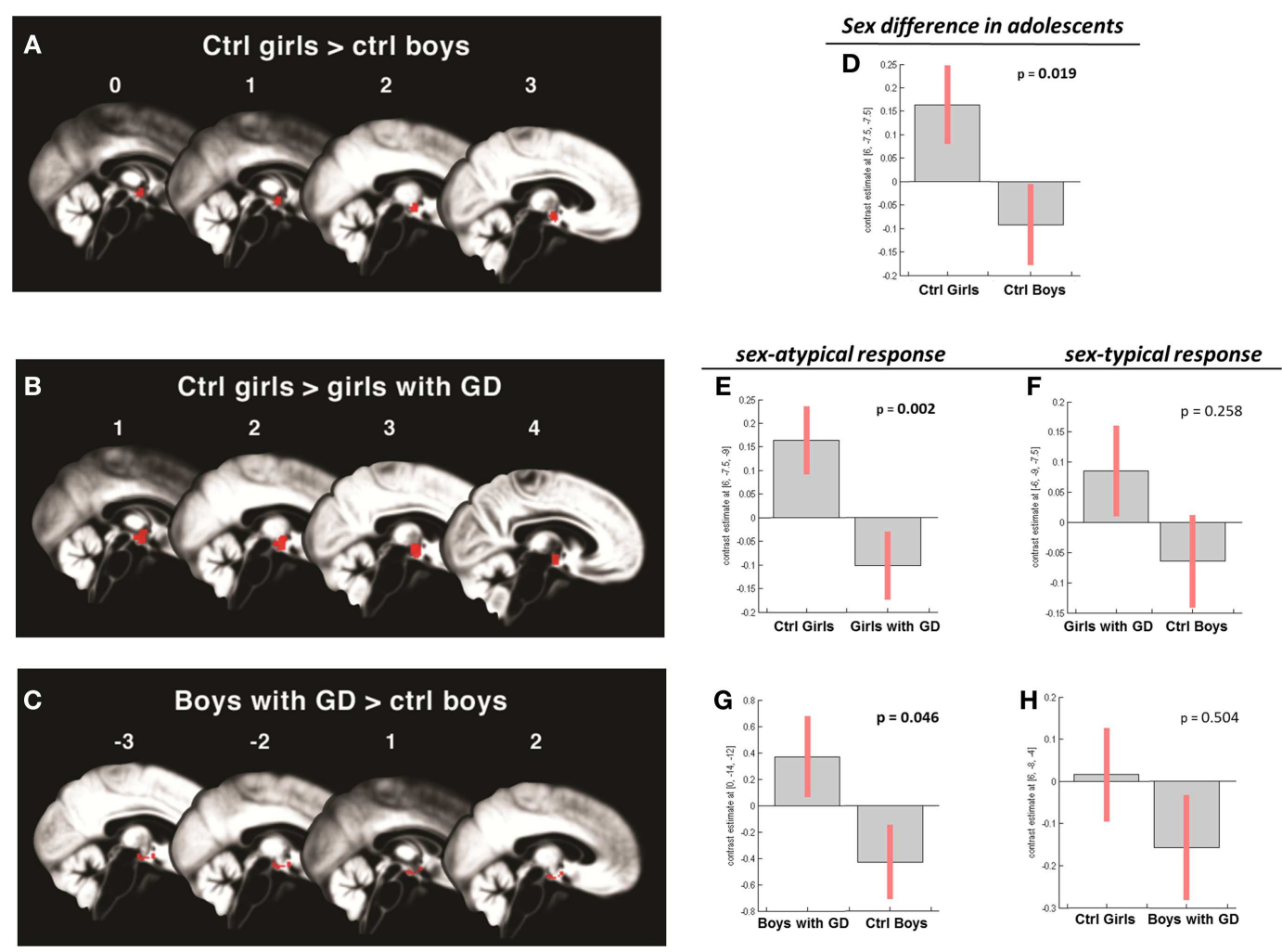

FIGURE 3 | (A-C) Show the anatomic locations of the significant hypothalamic activations, indicated in red, in response to the chemo-signal androstadienone in the adolescent age groups. The numbers above each sagittal plane represent the $x$-axis coordinates in Montreal Neurological Institute space. All voxels within the hypothalamic region of interest, surviving the statistical threshold of $p<0.05$ (FWE-corrected) are shown. (D-H) Show the bar graphs of the corresponding group contrasts. (D-E) Show the significant group contrasts of the first-order time-modulation regressor, indicating sensitization to the steroid odor in control girls compared with (A) control boys and (E) girls with gender dysphoria. (F) Shows the non-significant sex-typical (female-like) response of girls with gender dysphoria. (G) Displays the significant female-typical response of the boys with gender dysphoria for the contrast of the condition $\mathrm{ON}>\mathrm{OFF}$, irrespective of any effects of time; $\mathbf{( H )}$ shows the non-significant male-typical response of boys with gender dysphoria. during puberty and the assumed resulting adaptation to their own body odors (20-23). These findings suggested that puberty plays an important role in the development of sex differences in the olfactory sensitivity to androstadienone (48). Therefore, we hypothesized that the hypothalamic response in males, in contrast to that in females, would similarly be subject to neuronal adaptation after repeated exposure to the steroid odor. However, our results suggested only slight neuronal adaptation effects in boys, their activation remained relatively stable throughout the scanning session; instead, we found that girls showed an increase in hypothalamic responsiveness during the course of the odor stimulation. In line with the idea that androstadienone may function as a male modulator chemo-signal, our findings thus suggest that androstadienone indeed affects brain functions in females. Moreover, we showed that the sex difference in hypothalamic activation was not related to any hormonal or sexual maturation-related changes during puberty. Therefore, sex differences in neural responsiveness are probably not associated with differences in olfactory sensitivity to androstadienone and thus, its subjectively perceived intensity. Accordingly, we did not find any significant sex differences in the reported intensity ratings of androstadienone in both the pre-pubertal and adolescent groups.

\section{GENDER IDENTITY AND HYPOTHALAMIC ACTIVATION}

Gender dysphoria has been hypothesized to develop due to an altered sexual differentiation of the body and the brain during early development $(30,31)$. Here, we investigated a unique data set of individuals with GD at two different developmental stages, in order to determine whether they would respond to androstadienone in accordance with their natal sex, rather than their experienced gender. We found that both, adolescent girls and boys with GD showed hypothalamic activations that reflected their experienced gender. The sex difference that we observed in the control groups, was absent in both age groups of boys and girls diagnosed with GD. However, pre-pubertal girls with GD showed no differences in hypothalamic activation compared to both control boys and girls, whereas prepubertal boys with GD showed significant sex-typical hypothalamic activations and thus, in accordance with their natal sex. These findings thus suggest that individuals with GD possess certain functional brain characteristics, i.e., hypothalamic activation to androstadienone, of their experienced gender and thus that they may have undergone atypical neuronal sexual differentiation. However, this can only be observed reliably in adolescents with GD. 
Table 3 | ANOVA results showing sex-typical hypothalamic activations, reflecting their natal sex, in individuals with GD

\begin{tabular}{|c|c|c|c|c|c|c|c|}
\hline & Effect & $Z_{\max }$ & $x$ & $y$ & $z$ & $N$ & $P_{\mathrm{FWE}}$ \\
\hline \multicolumn{8}{|c|}{ GIRLS WITH GD > CTRL BOYS } \\
\hline Children & No effects & & & & & & \\
\hline \multirow[t]{4}{*}{ Adolescents } & Gender & - & - & - & - & - & - \\
\hline & Odor & 2.5 & 6 & -9 & -5 & 25 & 0.203 \\
\hline & ON-OFF & 1.7 & -6 & -7 & -5 & 2 & 0.512 \\
\hline & $\mathrm{ON}_{\mathrm{TM}}-\mathrm{OFF} \mathrm{T}_{\mathrm{TM}}$ & 2.3 & -6 & -9 & -8 & 25 & 0.258 \\
\hline \multicolumn{8}{|c|}{ CTRL GIRLS > BOYS WITH GD } \\
\hline \multirow[t]{4}{*}{ Children } & Gender & 3.0 & -6 & -10 & -6 & 35 & 0.065 \\
\hline & Odor & 2.6 & 3 & -16 & -9 & 27 & 0.168 \\
\hline & ON-OFF & 1.6 & -2 & -16 & -6 & 4 & 0.550 \\
\hline & $\mathrm{ON}_{\mathrm{TM}}-\mathrm{OFF} \mathrm{F}_{\mathrm{TM}}$ & 3.5 & -6 & -10 & -6 & 126 & 0.013 \\
\hline \multirow[t]{5}{*}{ Adolescents } & Gender & 2.2 & -2 & -4 & -10 & 5 & 0.336 \\
\hline & Odor & 1.5 & 2 & -14 & -12 & 4 & 0.699 \\
\hline & ON-OFF & - & - & - & - & - & - \\
\hline & ONTM-OFFTM & 1.7 & 6 & -8 & -4 & 7 & 0.504 \\
\hline & & 1.6 & -6 & -12 & -8 & 16 & 0.553 \\
\hline \multicolumn{8}{|c|}{ GIRLS WITH GD > BOYS WITH GD } \\
\hline Children & No effects & & & & & & \\
\hline \multirow[t]{4}{*}{ Adolescents } & Gender & - & - & - & - & - & - \\
\hline & Odor & 1.9 & 3 & -12 & -9 & 12 & 0.535 \\
\hline & ON-OFF & - & - & - & - & - & - \\
\hline & $\mathrm{ON}_{\mathrm{TM}}-\mathrm{OFF} \mathrm{F}_{\mathrm{TM}}$ & 1.9 & -6 & -7 & -11 & 1 & 0.459 \\
\hline
\end{tabular}

Ctrl, control; GD, gender dysphoria; $Z_{\max }, z$-value of peak activation voxel; $x$ y $z$, coordinates in Montreal Neurological Institute (MNI) space; N, number of clustering voxels (cluster size); FWE, family-wise error; the effects "Gender" and "Odor" indicate omnibus F-contrasts for the factors gender and odor stimulation; "ONOFF" and "ON $N_{T M}-O F F_{T M}$ " indicate directional $t$-contrasts for the condition effect and the first-order time-modulation regressor, respectively. Bold font indicates statistically significant effects.

\section{Girls diagnosed with GD}

Pre-pubertal girls with GD did not differ in hypothalamic activation from control girls indicating that they did not show a male-typical response. However, they also did not show a femaletypical hypothalamic activation, when compared to control boys, indicating that they did not differ from either of the control groups. It is possible that pre-pubertal girls with GD constitute a rather heterogeneous group with respect to future persisting GD. It has been shown that only about $15.8 \%$ of the childhood GD cases will eventually lead to adult GD (49). However, women present more often early-onset cases (50) and girls have overall higher persistency rates of GD into adolescence compared to boys (51). Thus, our finding that pre-pubertal girls with GD did not show a clearcut female- or male-typical response to androstadienone awaits further confirmation in the future, when it will be known who of our participants showed persisting GD into adolescence and adulthood.

The comparison of adolescent female controls versus adolescent girls with GD revealed very similar results as the control group comparisons, i.e., stronger hypothalamic activations in those subjects experiencing a female gender identity, and this effect was mainly driven by effects over time, reflecting sensitization.
Thus, adolescent girls with GD responded remarkably like their experienced gender (control boys). While speculative, these findings fit with the idea that this group of girls with GD (who are more homogenous in terms of future persisting GD compared to the pre-pubertal groups) may have had a more maletypical perinatal hormonal environment, resulting in the development of certain typically male functional sex characteristics of the brain. The hypogonadal state of adolescent girls with GD using GnRHa at the moment of data acquisition is not likely to account for these findings, since our results in the prepubertal control groups suggest that hypothalamic responsiveness to androstadienone is probably independent of puberty, and thus not affected by circulating sex hormones. Furthermore, animal studies showed that sexually dimorphic responses to volatile urinary odors were not dependent on circulating sex steroids, but rather developed under the influence of organizational effects of sex hormones $(52,53)$.

It should be noted, though, that sexual orientation of the participants with GD might present a confounding factor. Berglund et al. (27) showed that hypothalamic responsiveness to androstadienone in lesbian women was comparable to that of heterosexual men. In the literature, the majority of natal females with GD are reported to be gynephilic $(50,54)$, which was true as well for our group of adolescent gender dysphoric girls. Therefore, it cannot be ruled out that the resemblance in hypothalamic activation with the control boys might be due to their shared sexual orientation rather than their shared gender identity.

\section{Boys diagnosed with GD}

The hypothalamic response to androstadienone in the prepubertal boys with GD was not significantly stronger than that shown by the pre-pubertal control boys. Moreover, the reverse contrast (control girls $>$ boys with GD) revealed that pre-pubertal control girls showed significantly stronger hypothalamic activation, implying that boys with GD responded according to their natal sex. Again, it is likely that the younger, pre-pubertal sample of boys with GD constitutes a rather heterogeneous group in terms of future persistence of their gender dysphoric feelings (49, 51). Therefore, they may show more variability in hypothalamic responsiveness to androstadienone.

Adolescent boys with GD showed significantly stronger, thus sex-atypical hypothalamic activations, compared to control boys, although this effect was not modulated by any effects of time, thus sensitization to androstadienone, as we did observe in the control girls. Accordingly, the reverse comparison (control girls $>$ boys with GD) revealed no significant effects. The female-typical activation in adolescent boys with GD is in line with a previous study by Berglund et al. (26), who reported that adult men with GD differed significantly from a group of male controls in terms of hypothalamic activation during exposure to androstadienone. Their adult participants with GD were matched to control men with regard to hormonal status and gynephilic sexual orientation, whereas in our study adolescent boys with GD received GnRHa at the moment of data acquisition and the majority of our participants reported to have an androphilic sexual orientation. Thus, despite differences with regard to the participants' age, hormonal status, and sexual orientation, the findings of both studies suggest 
that males with GD possess certain female-typical brain functions, and may therefore have undergone sex-atypical early brain development.

Some limitations of the present study should be mentioned and may be addressed in future research. In most comparisons, the gender differences in hypothalamic response to androstadienone showed relatively small effect sizes. These effect sizes, in combination with relatively small group sizes (especially that of the pre-pubertal girls with GD with $N=17$ ), suggest that a lack of statistical power may be related to our failure in finding any significant effects for particularly this group. Therefore, additional analyses in other functional and structural (e.g., gray and white matter volumes or diffusion tensor imaging data) MRI measures, which are in preparation, should corroborate the present preliminary findings. Sexual orientation was difficult to assess in our young groups of participants. We therefore estimated their (future) sexual orientation by asking whether he/she had ever been in love. However, any relationship between sexual orientation and the response to androstadienone could not reliably be investigated in our groups.

In summary, the present study is the first to demonstrate that sex differences in hypothalamic activation upon smelling the chemo-signal androstadienone are not acquired during sexual maturation, under the influence of gonadal hormones during puberty, but may be considered hard-wired responses, which already can be observed in pre-pubertal children. Moreover, the current study is the first to explore sex-atypical hypothalamic responses to androstadienone in male and female individuals with GD at two different developmental stages. Our results indeed suggest that individuals with GD possess certain functional brain characteristics of their experienced gender and may have undergone atypical neuronal sexual differentiation.

\section{AUTHOR CONTRIBUTIONS}

Julie Bakker and Sarah M. Burke designed the study set-up. Sarah M. Burke performed the neuroimaging experiments, conducted the data analyses with advice of Dick J. Veltman, and wrote the manuscript. Daniel T. Klink performed the clinical assessments. Peggy T. Cohen-Kettenis, Dick J. Veltman, and Julie Bakker supervised the project. All authors contributed to interpretation of the data and revisions of the manuscript.

\section{ACKNOWLEDGMENTS}

We thank Prof. Thomas Hummel and his Smell and Taste lab at the University Medical School in Dresden (Germany) for his hospitality and the kind introduction into the field of olfactory fMRI. We thank Paul Groot from the Academic Medical Centre Amsterdam for his help with the data analyses, and we thank Prof Michael Baum from Boston University for his helpful comments on earlier versions of this manuscript. Last but not least, we are especially thankful to Dipl.-Ing. Johannes Burke, who developed and built the olfactometer equipment and programed the application software. This study was funded by a VICI grant (453-08-003) from the Dutch Science Foundation (Nederlandse Organisatie voor Wetenschappelijk Onderzoek) to Dr. Julie Bakker. Dr. Julie Bakker is a senior research associate of the Belgian Fonds National de la Recherche Scientifique.

\section{REFERENCES}

1. Kwan TK, Kraevskaya MA, Makin HL, Trafford DJ, Gower DB. Use of gas chromatographic-mass spectrometric techniques in studies of androst-16ene and androgen biosynthesis in human testis; cytosolic specific binding of 5alpha-androst-16-en-3-one. J Steroid Biochem Mol Biol (1997) 60:137-46. doi:10.1016/S0960-0760(96)00162-8

2. Nixon A, Mallet AI, Gower DB. Simultaneous quantification of five odorous steroids (16-androstenes) in the axillary hair of men. J Steroid Biochem (1988) 29:505-10. doi:10.1016/0022-4731(88)90185-9

3. Brooksbank BW, Cunningham AE, Wilson DA. The detection of androsta4,16-dien-3-one in peripheral plasma of adult men. Steroids (1969) 13:29-50. doi:10.1016/S0039-128X(69)80058-9

4. Kwan TK, Trafford DJ, Makin HL, Mallet AI, Gower DB. GC-MS studies of 16androstenes and other C19 steroids in human semen. J Steroid Biochem Mol Biol (1992) 43:549-56. doi:10.1016/0960-0760(92)90243-C

5. Brooksbank BW, Wilson DA, MacSweeney DA. Fate of androsta-4,16-dien-3one and the origin of 3-hydroxy-5-androst-16-ene in man. J Endocrinol (1972) 52:239-51. doi:10.1677/joe.0.0520239

6. Gower DB, Ruparelia BA. Olfaction in humans with special reference to odorous 16-androstenes: their occurrence, perception and possible social, psychological and sexual impact. J Endocrinol (1993) 137:167-87. doi:10.1677/joe.0.1370167

7. Bensafi M, Brown WM, Khan R, Levenson B, Sobel N. Sniffing human sex-steroid derived compounds modulates mood, memory and autonomic nervous system function in specific behavioral contexts. Behav Brain Res (2004) 152:11-22. doi:10.1016/j.bbr.2003.09.009

8. Jacob S, McClintock MK. Psychological state and mood effects of steroidal chemosignals in women and men. Horm Behav (2000) 37:57-78. doi:10.1006/ hbeh.1999.1559

9. Jacob S, Hayreh DJ, McClintock MK. Context-dependent effects of steroid chemosignals on human physiology and mood. Physiol Behav (2001) 74:15-27. doi:10.1016/S0031-9384(01)00537-6

10. Villemure C, Bushnell MC. The effects of the steroid androstadienone and pleasant odorants on the mood and pain perception of men and women. Eur J Pain (2007) 11:181-91. doi:10.1016/j.ejpain.2006.02.005

11. Bensafi M, Tsutsui T, Khan R, Levenson RW, Sobel N. Sniffing a human sexsteroid derived compound affects mood and autonomic arousal in a dosedependent manner. Psychoneuroendocrinology (2004) 29:1290-9. doi:10.1016/j. psyneuen.2004.03.007

12. Bensafi M, Brown WM, Tsutsui T, Mainland JD, Johnson BN, Bremner EA, et al. Sex-steroid derived compounds induce sex-specific effects on autonomic nervous system function in humans. Behav Neurosci (2003) 117:1125-34. doi:10.1037/0735-7044.117.6.1125

13. Grosser BI, Monti-Bloch L, Jennings-White C, Berliner DL. Behavioral and electrophysiological effects of androstadienone, a human pheromone. Psychoneuroendocrinology (2000) 25:289-99. doi:10.1016/S0306-4530(99)00056-6

14. Lundstöm J, Lundström JN, Gonçalves M, Esteves F, Olsson MJ. Psychological effects of subthreshold exposure to the putative human pheromone 4,16-androstadien-3-one. Horm Behav (2003) 44:395-401. doi:10.1016/j.yhbeh. 2003.06.004

15. Lundström JN, Olsson MJ. Subthreshold amounts of social odorant affect mood, but not behavior, in heterosexual women when tested by a male, but not a female, experimenter. Biol Psychol (2005) 70:197-204. doi:10.1016/j.biopsycho. 2005.01.008

16. Wyart C, Webster WW, Chen JH, Wilson SR, McClary A, Khan RM, et al. Smelling a single component of male sweat alters levels of cortisol in women. J Neurosci (2007) 27:1261-5. doi:10.1523/JNEUROSCI.4430-06.2007

17. Parma V, Tirindelli R, Bisazza A, Massaccesi S, Castiello U. Subliminally perceived odours modulate female intrasexual competition: an eye movement study. PLoS One (2012) 7:e30645. doi:10.1371/journal.pone.0030645

18. Savic I, Berglund H, Gulyas B, Roland P. Smelling of odorous sex hormonelike compounds causes sex-differentiated hypothalamic activations in humans. Neuron (2001) 31:661-8. doi:10.1016/S0896-6273(01)00390-7

19. Burke SM, Veltman DJ, Gerber J, Hummel T, Bakker J. Heterosexual men and women both show a hypothalamic response to the chemo-signal androstadienone. PLoS One (2012) 7:e40993. doi:10.1371/journal.pone.0040993

20. Chopra A, Baur A, Hummel T. Thresholds and chemosensory event-related potentials to malodors before, during, and after puberty: differences related to sex and age. Neuroimage (2008) 40:1257-63. doi:10.1016/j.neuroimage.2008. 01.015 
21. Dorries KM, Schmidt HJ, Beauchamp GK, Wysocki CJ. Changes in sensitivity to the odor of androstenone during adolescence. Dev Psychobiol (1989) 22:423-35. doi:10.1002/dev.420220502

22. Hummel T, Krone F, Lundström JN, Bartsch O. Androstadienone odor thresholds in adolescents. Horm Behav (2005) 47:306-10. doi:10.1016/j.yhbeh.2004. 10.007

23. Koelega HS, Köster EP. Some experiments on sex differences in odor perception. Ann N Y Acad Sci (1974) 237:234-46. doi:10.1111/j.1749-6632.1974.tb49859.x

24. Schulz KM, Molenda-Figueira H, Sisk CL. Back to the future: the organizationalactivational hypothesis adapted to puberty and adolescence. Horm Behav (2009) 55:597-604. doi:10.1016/j.yhbeh.2009.03.010

25. Sisk CL, Zehr JL. Pubertal hormones organize the adolescent brain and behavior. Front Neuroendocrinol (2005) 26:163-74. doi:10.1016/j.yfrne.2005.10.003

26. Berglund H, Lindström P, Dhejne-Helmy C, Savic I. Male-to-female transsexuals show sex-atypical hypothalamus activation when smelling odorous steroids. Cereb Cortex (2008) 18:1900-8. doi:10.1093/cercor/bhm216

27. Berglund H, Lindström P, Savic I. Brain response to putative pheromones in lesbian women. Proc Natl Acad Sci U S A (2006) 103:8269-74. doi:10.1073/pnas. 0600331103

28. Savic I, Berglund H, Lindström P. Brain response to putative pheromones in homosexual men. Proc Natl Acad Sci U S A (2005) 102:7356-61. doi:10.1073/ pnas.0407998102

29. American Psychiatric Association. Diagnostic and Statistical Manual of Mental Disorders, Fifth Edition (DSM-5). Arlington, VA: American Psychiatric Association (2013).

30. Swaab DF, Garcia-Falgueras A. Sexual differentiation of the human brain in relation to gender identity and sexual orientation. Funct Neurol (2009) 24:17-28.

31. van Goozen SH, Gooren LJG, Sanders G, Cohen-Kettenis PT, Slabbekoorn D. Organizing and activating effects of sex hormones in homosexual transsexuals. Behav Neurosci (2002) 116:982-8. doi:10.1037/0735-7044.116.6.982

32. Marantz S, Coates S. Mothers of boys with gender identity disorder: a comparison of matched controls. J Am Acad Child Adolesc Psychiatry (1991) 30:310-5. doi:10.1097/00004583-199103000-00022

33. Zucker KJ, Bradley SJ. Gender Identity Disorder and Psychosexual Problems in Children and Adolescents. New York, NY: The Guilford Press (1995).

34. Coolidge FL, Thede LL, Young SE. The heritability of gender identity disorder in a child and adolescent twin sample. Behav Genet (2002) 32:251-7. doi:10.1023/A:1019724712983

35. Gooren L. The biology of human psychosexual differentiation. Horm Behav (2006) 50:589-601. doi:10.1016/j.yhbeh.2006.06.011

36. Kreukels BPC, Cohen-Kettenis PT. Puberty suppression in gender identity disorder: the Amsterdam experience. Nat Rev Endocrinol (2011) 7:466-72. doi: $10.1038 /$ nrendo.2011.78

37. Delemarre-van de Waal HA, Cohen-Kettenis PT. Clinical management of gender identity disorder in adolescents: a protocol on psychological and paediatric endocrinology aspects. Eur J Endocrinol (2006) 155:S131-7. doi:10.1530/eje.1. 02231

38. Hembree WC, Cohen-Kettenis P, Delemarre-van de Waal HA, Gooren LJ, Meyer WJ, Spack NP, et al. Endocrine treatment of transsexual persons: an Endocrine Society clinical practice guideline. J Clin Endocrinol Metab (2009) 94:3132-54. doi:10.1210/jc.2009-0345

39. Marshall WA, Tanner JM. Variations in the pattern of pubertal changes in boys. Arch Dis Child (1970) 45:13-23. doi:10.1136/adc.45.239.13

40. Marshall WA, Tanner JM. Variations in pattern of pubertal changes in girls. Arch Dis Child (1969) 44:291-303. doi:10.1136/adc.44.235.291

41. Hummel T, Kobal G, Gudziol H, Mackay-Sim A. Normative data for the "Sniffin' sticks" including tests of odor identification, odor discrimination, and olfactory thresholds: an upgrade based on a group of more than 3,000 subjects. Eur Arch Otorhinolaryngol (2007) 264:237-43. doi:10.1007/s00405-006-0173-0

42. Kobal G, Klimek L, Wolfensberger M, Gudziol H, Temmel A, Owen CM, et al. Multicenter investigation of 1,036 subjects using a standardized method for the assessment of olfactory function combining tests of odor identification odor discrimination, and olfactory thresholds. Eur Arch Otorhinolaryngol (2000) 257:205-11. doi:10.1007/s004050050223

43. Kobal G, Hummel T, Sekinger B, Barz S, Roscher S, Wolf S. "Sniffin' sticks”: screening of olfactory performance. Rhinology (1996) 34:222-6.

44. Lemieux L, Salek-Haddadi A, Lund TE, Laufs H, Carmichael D. Modelling large motion events in fMRI studies of patients with epilepsy. Magn Reson Imaging (2007) 25:894-901. doi:10.1016/j.mri.2007.03.009

45. Brett M, Anton J, Valabregue R, Poline J-B. Region of interest analysis using an SPM toolbox [abstract]. Presented at the 8th International Conference on Functional Mapping of the Human Brain. Sendai (2002).

46. Makris N, Swaab DF, van der Kouwe A, Abbs B, Boriel D, Handa RJ, et al. Volumetric parcellation methodology of the human hypothalamus in neuroimaging: normative data and sex differences. Neuroimage (2013) 69:1-10. doi:10.1016/j.neuroimage.2012.12.008

47. Baroncini M, Jissendi P, Balland E, Besson P, Pruvo J-P, Francke J-P, et al. MRI atlas of the human hypothalamus. Neuroimage (2012) 59:168-80. doi:10.1016/ j.neuroimage.2011.07.013

48. Lundström JN, Hummel T, Olsson MJ. Individual differences in sensitivity to the odor of 4,16-androstadien-3-one. Chem Senses (2003) 28:643-50. doi:10.1093/chemse/bjg057

49. Steensma TD, Biemond R, de Boer F, Cohen-Kettenis PT. Desisting and persisting gender dysphoria after childhood: a qualitative follow-up study. Clin Child Psychol Psychiatry (2011) 16:499-516. doi:10.1177/1359104510378303

50. Nieder TO, Herff M, Cerwenka S, Preuss WF, Cohen-Kettenis PT, De Cuypere $\mathrm{G}$, et al. Age of onset and sexual orientation in transsexual males and females. J Sex Med (2011) 8:783-91. doi:10.1111/j.1743-6109.2010.02142.x

51. Steensma TD, McGuire JK, Kreukels BPC, Beekman AJ, Cohen-Kettenis PT. Factors associated with desistence and persistence of childhood gender dysphoria: a quantitative follow-up study. J Am Acad Child Adolesc Psychiatry (2013) 52:582-90. doi:10.1016/j.jaac.2013.03.016

52. Baum MJ, Keverne EB. Sex difference in attraction thresholds for volatile odors from male and estrous female mouse urine. Horm Behav (2002) 41:213-9. doi:10.1006/hbeh.2001.1749

53. Pierman S, Douhard Q, Balthazart J, Baum MJ, Bakker J. Attraction thresholds and sex discrimination of urinary odorants in male and female aromatase knockout (ArKO) mice. Horm Behav (2006) 49:96-104. doi:10.1016/j.yhbeh. 2005.05.007

54. Lawrence AA. Sexual orientation versus age of onset as bases for typologies (subtypes) for gender identity disorder in adolescents and adults. Arch Sex Behav (2010) 39:514-45. doi:10.1007/s10508-009-9594-3

55. Gläscher, J. Visualization of group inference data in functional neuroimaging. Neuroinformatics (2009) 7:73-82. doi:10.1007/s12021-008-9042-x

Conflict of Interest Statement: The authors declare that the research was conducted in the absence of any commercial or financial relationships that could be construed as a potential conflict of interest.

Received: 21 November 2013; accepted: 10 April 2014; published online: 28 May 2014. Citation: Burke SM, Cohen-Kettenis PT, Veltman DJ, Klink DT and Bakker J (2014) Hypothalamic response to the chemo-signal androstadienone in gender dysphoric children and adolescents. Front. Endocrinol. 5:60. doi: 10.3389/fendo.2014.00060

This article was submitted to Neuroendocrine Science, a section of the journal Frontiers in Endocrinology.

Copyright ( $) 2014$ Burke, Cohen-Kettenis, Veltman, Klink and Bakker. This is an openaccess article distributed under the terms of the Creative Commons Attribution License (CC BY). The use, distribution or reproduction in other forums is permitted, provided the original author(s) or licensor are credited and that the original publication in this journal is cited, in accordance with accepted academic practice. No use, distribution or reproduction is permitted which does not comply with these terms. 